\title{
Darunavir Resistance in HIV Infecting Protease Inhibitor-Experienced Mexican Patients*
}

\author{
Carlos A. Agudelo ${ }^{1}$, Luis E. Soto-Ramírez ${ }^{1}$, Abraham Katime-Zúñiga ${ }^{1}$, Lorena Cabrera-Ruíz ${ }^{2}$, \\ Hugo Lara-Sánchez ${ }^{3}$, Juan J. Calva ${ }^{3 \#}$ \\ ${ }^{1}$ Department of Infectious Diseases, “Instituto Nacional de Ciencias Médicas y Nutrición Salvador Zubirán”, Mexico City, Mexico; \\ ${ }^{2}$ Department of Infectious Diseases, Hospital Médica Sur and GUIAR Group, Mexico City, Mexico; ${ }^{3}$ The Clinical Epidemiology \\ Unit, "Instituto Nacional de Ciencias Médicas y Nutrición Salvador Zubirán”, Mexico City, Mexico. \\ Email: juanjcalva@gmail.com
}

Received July $9^{\text {th }}, 2013$; revised August $9^{\text {th }}, 2013$; accepted August $19^{\text {th }}, 2013$

Copyright (C) 2013 Carlos A. Agudelo et al. This is an open access article distributed under the Creative Commons Attribution License, which permits unrestricted use, distribution, and reproduction in any medium, provided the original work is properly cited.

\begin{abstract}
Background: Darunavir (DRV) is a useful antiretroviral treatment in the salvage therapy of multiclass-resistant HIVinfected patients. This study's aim was to determine the frequency and risk factors for DRV resistance-associated mutations (DRV-RAM) among DRV-naïve Mexican patients with virologic failure after extensive antiretroviral treatment and exposure to at least one protease inhibitor (PI). Methods: HIV-infected patients with a history of at least 2 failed regimes were included and their clinical histories and genotype resistance tests were analyzed. Major PI resistance-associated mutations (PI-RAM), DRV-RAM and resistance to DRV were defined according to the IAS-USA criteria. Previous exposure to PI was compared between patients with DRV-resistant HIV and DRV-susceptible HIV-infected controls. Results: The median number of major PI-RAM was $2(\mathrm{IQR}=0-3)$. In $54.7 \%(95 \% \mathrm{CI}=50.0 \%-59.4 \%)$ of 631 subjects, no DRV-RAM were found on viral genotyping and $6.7 \%(95 \% \mathrm{CI}=4.8 \%-8.6 \%)$ had 3 or more DRV-RAM. The two most frequently found DRV-RAM were in codons I84V (in $22.7 \%$ of cases) and L33F (in $20 \%$ of cases) in the viral protease gene. The number of major PI-RAM (as a surrogate marker of duration and number of PI used) and previous exposure to (fos) amprenavir or tipranavir were independently associated with DRV-resistant HIV infection. Conclusions: In this Mexican population, despite a high prior PI exposure, HIV-DRV resistance rate is relatively low and successful viral control with DRV-containing combined salvage therapy is expected in most patients.
\end{abstract}

Keywords: Darunavir; Resistance; Risk Factors; Prevalence; Mexico

\section{Introduction}

Randomized clinical trials have shown that ritonavirboosted darunavir (DRV/r) as a component of salvage regimens (and at least one other fully active drug), leads to significantly higher rates of lasting virological control when compared with conventional protease inhibitors (PI), among patients infected with multidrug-resistant HIV and an extensive treatment history [1-6].

Eleven specific darunavir resistance-associated mutations (DRV-RAM) in the viral protease gene have been linked to decrease in vitro HIV susceptibility as well as sub-optimal clinical responses to darunavir [7]. A loss of

\footnotetext{
*Author Disclosure: L. E. S-R. has received payment for lectures from MSD, Janssen-Cilag and ViiV. J. J. C. has served as a Board member of the DSMB of the CADIRIS Study. None of the other authors had conflicts of interest or funding sources.

${ }^{\#}$ Corresponding author.
}

response begins to occur with one mutation but 3 or more mutations and additional numerous protease inhibitor (PI) resistance-associated mutations (PI-RAM) lead to a greatly diminished virologic suppression rate. Participants in the POWER 1 and 2 trials (in the DRV/r arm) as well as the DUET- 1 and DUET-2 studies, had a baseline prevalence of 3 or more DRV-RAM of $22 \%, 41 \%$ and $44 \%$, respectively [2-5].

In routine clinical practice, the rate of occurrence of these 11 DRV-RAM in PI-treated patients not participating in clinical trials, has been reported in some populations [8-10]. A low percentage (ranging between 4.1\% and $6.7 \%$ ) of individuals with 3 or more DRV-RAM, has consistently been found in these surveys. Furthermore, these studies have shown an association between the number of prior PI used, the total number of PI resistance mutations, previous treatment with (fos) amprenavir, and 
a greater risk of developing DRV-RAM.

In Mexico, scaling up of free access to antiretroviral treatment started approximately 15 years ago; during the first 4 years, about one third of total patient-months were treated with a PI (predominantly unboosted indinavir and unboosted saquinavir) [11]. Over the past few years, close to $29 \%$ of combined therapy prescriptions have included a PI with a broad range of agents, mostly: ritonavir-boosted lopinavir, ritonavir-boosted atazanavir, ritonavir-boosted saquinavir and ritonavir-boosted indinavir [12].

This study's aim was to assess the impact of this long and extensive use of diverse PI in our country, on the number of DRV-RAM in HIV infected DRV-naïve patients and virologic failure with a long-standing history of antiretroviral therapy with several regimens. Also, we determined whether the presence of 3 or more DRVRAM was associated with surrogate markers of the level of prior PI selective pressure.

\section{Methods}

\subsection{Study Population}

A retrospective, cross-sectional study was conducted to estimate the prevalence of HIV DRV-RAM and by casecontrol analysis, the association between certain characteristics in study patients and DRV-resistant HIV was assessed.

To be eligible, patients required: 1) to be considered as virologic failures (ongoing viral replication as defined by an HIV viral load above 50 copies/ $\mathrm{ml}$ in at least two consecutive measurements) and on antiretroviral therapy at the time of this survey, 2) to have a history of at least two antiretroviral failed regimes, 3) prior treatment with at least one PI, 4) no previous use of DRV, 5) an HIV genotype resistant test performed while receiving the last drug scheme, 6) at least one PI resistance mutation (PIRAM) in this genotyping and 7) complete clinical data. Between 2008 and 2010, patients were selected from two populations of cases whose physician had requested and received a recommendation to optimize the salvage regimen of heavily-treated experienced patients, by one of two national antiretroviral therapy peer-advisory committees. The Board for the rational use of antiretrovirals (CORESAR) provides advice to physicians caring for individuals in the Mexican Ministry of Health system; the Inter-institutional group for antiretroviral treatment (GUIAR) provides help to practitioners caring for patients in this health system or in two of the largest national social security systems (IMSS and ISSSTE).

Demographic, virologic, prior specific drug exposure and drug regimen at the time of last failure data were recorded in each case.

\subsection{Drug Resistance Testing}

Genetic sequencing of protease and reverse transcriptase HIV genes was conducted in plasma, using the Viroseq ${ }^{\circledR}$, HIV-1 kit (Abbott Laboratories, Abbott Park, Illinois) in samples from the cases in CORESAR, and the Trugene ${ }^{\circledR}$, HIV-1 kit (Siemens, Erlangen, Germany) in samples from GUIAR. Drug resistance mutations within the pol gene were interpreted following the 2011 International Antiviral Society-USA panel list. Accordingly, the following 11 resistance mutations were evaluated for darunavir: V11I, V32I, L33F, I47V, I50V, I54L/M, T74P, L76V, I84V and L89V [13]. Darunavir resistance was defined as the presence of 3 or more of these mutations.

\subsection{Statistical Analysis}

All data are reported as absolute numbers and percentages, as well as medians (Md) and interquartile ranges (IQR). Comparisons between 2 independent groups were established using the U-Mann-Whitney test for continuous variables and the Pearson $\chi^{2}$ or the Fisher exact test for categorical variables. Comparison of dimensional data distribution across more than 2 independent groups, was analyzed with the Kruskal-Wallis non-parametric 1-way ANOVA test. Univariate and multivariate logistic regression analysis were performed to assess factors associated to the presence of each DRV-RAM and DRV resistance. The magnitude of the association was expressed as the odds ratio (OR) and its 95\% confidence interval (95\% CI). Statistical significance was reflected by a $\mathrm{P}$ value < 0.05. Association between continuous variables was measured with the Pearson correlation coefficient (r). A receiver operating-characteristic (ROC) curve was plotted to assess the diagnostic performance of the number of major PI-RAM in the prediction of darunavir-resistance. Statistical analysis was carried out using the $\operatorname{SPSS}^{\circledR} 16.0$ (IBM Corp. Armonk, NY).

\subsection{Ethical Considerations}

This study was approved by the Research Ethics Committee of the "Instituto Nacional de Ciencias Médicas y Nutrición Salvador Zubirán” (Ref \#62), and was conducted in accordance with The Declaration of Helsinki.

\section{Results}

\subsection{Study Population}

From a total of 868 referred cases (476 patients in CORESAR and 392 patients in GUIAR), 631 (73\%) met the inclusion criteria. Study patient features are depicted in Table 1. Most had received various combinations of antiretroviral therapies without optimal viral control, over several years. 
Table 1. Characteristics of the 631 study subjects.

\begin{tabular}{|c|c|}
\hline Age (years): Md (IQR) & $40(33-46)$ \\
\hline Duration since HIV diagnosis (years): Md (IQR) & $9(6-11.6)$ \\
\hline Nadir T-CD4+ cell count (cells $\left./ \mathrm{mm}^{3}\right):$ Md (IQR) & $61(23-155)$ \\
\hline Patients with no documented HIV plasma viral load under the limit of detection at any time: $\mathrm{n}(\%)$ & $328(52)$ \\
\hline Duration with ARV therapy (years): Md (IQR) & $8(5-11)$ \\
\hline Number of prior drug regimens: $\mathrm{Md}(\mathrm{IQR})$ & $4(3-5)$ \\
\hline Number of PI previously used: Md (IQR) & $2(2-3)$ \\
\hline Prior use of (fos) amprenavir: $\mathrm{n}(\%)$ of cases & $40(6.3)$ \\
\hline Prior use of tipranavir: $\mathrm{n}(\%)$ of cases & $98(15.5)$ \\
\hline Duration of prior use of any PI (years): Md (IQR) & $5(2.9-7.7)$ \\
\hline Plasma HIV viral load prior to genotyping ( $\left.\log _{10} \mathrm{cop} / \mathrm{ml}\right)$ : Md (IQR) & $4.52(3.95-5)$ \\
\hline T-CD4+ cell count prior to genotyping $\left(\right.$ cells/mm $\left./ \mathrm{mm}^{3}\right): \mathrm{Md}(\mathrm{IQR})$ & $192(86.7-328.3)$ \\
\hline \multicolumn{2}{|l|}{ ARV regimen at time of genotyping: $n(\%)$ of cases } \\
\hline NRTI + PI & $429(68 \%)$ \\
\hline $\mathrm{NRTI}+\mathrm{NN}$ & $118(19 \%)$ \\
\hline $\mathrm{NRTI}+\mathrm{PI}+\mathrm{NN}$ & $31(5 \%)$ \\
\hline Other & $53(8 \%)$ \\
\hline Number of major PI-resistance associated mutations: Md (IQR) & $2(0-3)$ \\
\hline
\end{tabular}

$\mathrm{NRTI}=$ Nucleos $(\mathrm{t})$ ide analogue reverse transcriptase inhibitor; PI = Protease inhibitor; $\mathrm{NN}=$ Non-nucleoside reverse transcriptase inhibitor.

\subsection{Prevalence of Major PI-RAM and DRV-RAM}

Table 2 shows the distribution of the 631 study patients according to the number of PI-RAM and DRV-RAM found in their HIV genotype resistance test. Two or more major PI-RAM and 4 or more major PI-RAM were identified in $60 \%$ and $18 \%$ of the patients, respectively. More than half $(54.7 \%, 95 \% \mathrm{CI}=49.5 \%-59.9 \%)$ of patients had no DRV-RAM; $20.9 \%$ (95\% CI $=14 \%-27.8 \%)$ had more than one DRV-RAM and 6.7\% (95\% CI $=4.7 \%$ $8.7 \%$ ) were infected with HIV resistant to darunavir (genotype with 3 or more DRV-RAM).

The two most frequently identified DRV-RAM in the overall population were mutation I84V (22.7\% of cases) and mutation L33F (20\% of cases). Other DRV-RAM was present in less than $10 \%$ of the overall population. Among the 42 patients with HIV resistant to DRV, the relative frequency of all DRV-RAM was: I84V (76.2\% of cases), L33F (54.8\% of cases) V32I (54.8\% of cases), I $47 \mathrm{~V}(47.6 \%$ of cases), L89V (31.0\% of cases), I54L (28.6\% of cases), T74P (21.4\% of cases), V11I (19.0\% of cases), I54M (16.7\% of cases), L76V (7.1\% of cases) and I50V (2.4\% of cases).

\subsection{Association between DRV-RAM and Prior Exposure to Other PI}

An association between the presence of a particular DRV-RAM and prior use of other specific PI was established. There was a significant association between (fos) amprenavir use (vs. no prior use of these PI) and the identification of the following five DRV-RAM: I54M $(\mathrm{OR}=13.9 ; 95 \% \mathrm{CI}=4.1-47.8), \mathrm{I} 50 \mathrm{~V}(\mathrm{OR}=6.2 ; 95 \%$ $\mathrm{CI}=1.1-32.8)$, V32I $(\mathrm{OR}=3.2 ; 95 \% \mathrm{CI}=1.2-8)$, $\mathrm{I} 47 \mathrm{~V}(\mathrm{OR}=3.1 ; 95 \% \mathrm{CI}=1.3-7.4)$ and $\mathrm{L} 33 \mathrm{~F}(\mathrm{OR}=2.3$; $95 \% \mathrm{CI}=1.2$ - 4.6). Moreover, there was also a significant association between tipranavir use (vs. no prior use of this PI) and the identification of the following five DRV-RAM: I84V $(\mathrm{OR}=4.9 ; 95 \% \mathrm{CI}=3.1-7.7)$, T74P $(\mathrm{OR}=4.0 ; 95 \% \mathrm{CI}=1.5-11), \mathrm{L} 33 \mathrm{~F}(\mathrm{OR}=3.1 ; 95 \% \mathrm{CI}$ $=1.9-4.9)$, L32I $(\mathrm{OR}=2.5 ; 95 \% \mathrm{CI}=1.2-5.2)$ and $\mathrm{I} 47 \mathrm{~V}(\mathrm{OR}=2.4 ; 95 \% \mathrm{CI}=1.2-4.7)$.

Patients infected with HIV and harboring 3 or more DRV-RAM compared with those with less than 3 DRVRAM, had been treated with a significantly greater number of PI and had a longer cumulative time period of protease inhibitor exposure; moreover, these individuals were significantly more likely to have used a regimen with two 
PI (independently of ritonavir use as a booster), (fos) amprenavir, lopinavir/ritonavir, and tipranavir (see Table 3). The median number of major PI-RAM was significantly greater in patients with 3 or more DRV-RAM.

A significant correlation between the number of major PI-RAM and the previous amount and duration of PI administration was also detected (Pearson correlation coefficients $=0.30, p<0.01$ and $0.23, p<0.01$, respectively). Hence, the number of major PI-RAM was used as a surrogate marker of the level of prior PI pharmacologic selective pressure. On multivariate analysis, prior use of (fos) amprenavir, the use of tipranavir and the number of major PI-RAM were independently associated with 3 or more DRV-RAM (see Table 4). There is a 3.7 fold increase in the risk of having a darunavir resistant HIV infection per one extra major PI-RAM.

As Figure 1 shows, the median number of DRV-RAM rose as the number of major PI-RAM increased (Pearson correlation coefficient $=0.73 ; \mathrm{P}<0.001$ ). The distribution of the number of DRV-RAM was significantly different across categories of the number of major PI-RAM
Table 2. Distribution of 631 cases according to the number of major protease inhibitor-resistance associated (PI-RAM) and darunavir-resistance associated mutations (DRV-RAM) in genotype testing.

\begin{tabular}{ccc}
\hline Number of RAM & \multicolumn{2}{c}{ Number (\%) of patient with } \\
\hline & Major PI-RAM & DRV-RAM \\
& $\mathrm{n}(\%)$ & $\mathrm{n}(\%)$ \\
0 & $185(29.3)$ & $345(54.7)$ \\
1 & $72(11.4)$ & $154(24.4)$ \\
2 & $115(18.2)$ & $90(14.3)$ \\
3 & $148(23.5)$ & $28(4.4)$ \\
4 & $70(11.1)$ & $5(0.8)$ \\
5 & $29(4.6)$ & $7(1.1)$ \\
6 & $9(1.4)$ & $2(0.3)$ \\
7 & $3(0.5)$ & $0(0)$ \\
& $631(100)$ & $631(100)$ \\
\hline
\end{tabular}

Table 3. Comparison of prior exposure to other protease inhibitors (PI) between subjects infected with HIV harboring 3 or more darunavir-resistance associated mutations (DRV-RAM) vs with less than 3 DRV-RAM.

\begin{tabular}{cccc}
\hline Variable & $\begin{array}{c}\text { HIV with 3 or more DRV-RAM } \\
(42 \text { subjects) }\end{array}$ & $\begin{array}{c}\text { HIV with less than 3 DRV-RAM } \\
\text { (589 subjects) }\end{array}$ & P value \\
\hline Number of PI used: median (IQR) & $3(2,4)$ & $2(1,3)$ & $<0.01$ \\
Duration of PI use (years): median (IQR) & $7.1(4.6,8.4)$ & $4.9(2.8,7.7)$ & 0.02 \\
(fos) amprenavir use: \% of patients & 24 & 5 & $<0.001$ \\
Tipranavir use: \% of patients & 40 & 65 & 0.001 \\
Lopinavir/ritonavir use: \% of patients & 81 & 14 & 0.05 \\
Double PI use: \% of patients & 26 & $2(0,3)$ & $<0.001$ \\
Number of major PI-RAM*: median (IQR) & $5(3,6)$ & & \\
\hline
\end{tabular}

*Protease inhibitor resistance-associated mutation.

Table 4. Association between infection by HIV harboring 3 or more darunavir-resistance associated mutations (DRV-RAM) and prior exposure to other protease inhibitors (PI) in 631 subjects.

\begin{tabular}{|c|c|c|c|c|c|c|}
\hline \multirow[t]{2}{*}{ Variable } & \multicolumn{3}{|c|}{ Univariate } & \multicolumn{3}{|c|}{ Multivariate } \\
\hline & Odds ratio & $95 \% \mathrm{CI}$ & $\mathrm{p}$ value & Odds ratio & $95 \% \mathrm{CI}$ & $\mathrm{p}$ value \\
\hline Duration of PI use, per one year increase & 1.1 & $1.0-1.2$ & 0.03 & & & \\
\hline (fos) amprenavir use/no use & 5.8 & $2.6-12.9$ & $<0.001$ & 7.3 & $2.4-22$ & $<0.001$ \\
\hline Tipranavir use/no use & 4.3 & $2.2-8.2$ & $<0.001$ & 2.4 & $1.0-5.7$ & 0.04 \\
\hline Lopinavir/ritonavir use/no use & 2.3 & $1.0-5.0$ & 0.04 & 0.7 & $0.2-2.1$ & NS \\
\hline Double PI use/no use & 2 & $1.0-4.2$ & 0.05 & 1.5 & $0.6-4.3$ & NS \\
\hline Number of major PI-RAM ${ }^{*}$, per one mutation increase & 4.3 & $3.0-6.2$ & $<0.001$ & 4.7 & $3.0-7.1$ & $<0.001$ \\
\hline
\end{tabular}

* Protease inhibitor resistance-associated mutation. 


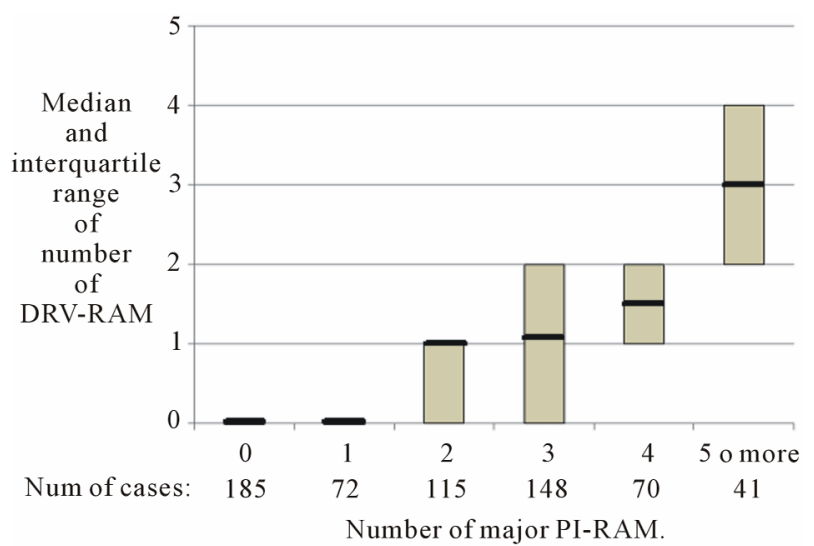

Figure 1. Distribution of the number of darunavir resistance-associated mutations (DRV-RAM) according to the number of major protease inhibitor resistance-associated mutations (PI-RAM) in genotype testing of 631 study subjects (Kruskal-Wallis test: $\mathbf{p}<\mathbf{0 . 0 0 1}$ ).

(Kruskal-Wallis test: $\mathrm{p}<0.001)$. The area under the curve of the ROC curve plotting the number of major PI-RAM as predictors of DRV-resistance was 0.90 . With a cut-off point of 6 or more major PI-RAM, we found a positive predictive value of $100 \%(95 \% \mathrm{CI}=73 \%$ $100 \%)$ and a negative predictive value of $95 \%(95 \% \mathrm{CI}=$ $93.3 \%$ - 96.7\%) in the diagnosis or DRV-resistance. Four non-DRV major PI-RAM were found to be significantly associated with darunavir resistance: $\mathrm{M} 46 \mathrm{I}(\mathrm{OR}=2.5$ $95 \% \mathrm{CI}=1.3-4.7 ; \mathrm{p}<0.01), \mathrm{Q} 58 \mathrm{E}(\mathrm{OR}=3.195 \% \mathrm{CI}=$ 1.5 - 6.4; $\mathrm{p}<0.01), \mathrm{V} 82 \mathrm{~T}(\mathrm{OR}=2.795 \% \mathrm{CI}=1.1-6.4$; $\mathrm{p}=0.03)$ and $\mathrm{L} 90 \mathrm{M}(\mathrm{OR}=3.095 \% \mathrm{CI}=1.5-6.0 ; \mathrm{p}<$ $0.01)$.

\subsection{Other Factors Associated with Genotypic Resistance to Darunavir}

Comparison of demographic and clinical characteristics between patients infected with darunavir-resistant HIV versus darunavir-susceptible HIV, showed that the former had a higher viral load at the time of resistance testing (median HIV-RNA $=93,253\left[4.97 \log _{10}\right]$ copies $/ \mathrm{ml}$ vs. $30,150\left[4.48 \log _{10}\right]$ copies $/ \mathrm{ml}$, respectively; $\mathrm{p}=0.02$ ) and a longer-lasting virological failure with the last administered regimen (median of 41.8 months vs. 30.3 months, respectively; $\mathrm{p}=0.02$ ).

\section{Discussion}

Darunavir, a second-generation PI with a strong affinity for the HIV-1 protease and a relatively very high genetic barrier to resistance development, has become the standard-of-care PI in advanced salvage regimens [14]. Individuals in whom previous various PI-containing antiretroviral regimens have failed, are generally infected with HIV bearing multiple resistance mutations induced by conventional first generation PI and with potential cross- resistance with the new extended-activity PI, such as darunavir and/or tipranavir. In these circumstances, resistance testing is mandatory, particularly in populations where long-lasting and failing inappropriate combinations, including unboosted PI, have been used as has occurred in certain health facilities in Mexico [11,12]. A fundamental issue in the rational approach to patients infected with multidrug resistant HIV, is the knowledge of the pre-resistance test likelihood of finding DRV-RAM; this requires continuous monitoring of the rate of occurrence of these viral genetic determinants at the population level. Moreover, it is important to have the ability to predict who is at higher risk of infection with HIV with 3 or more DRV-RAM and therefore, have a lower probability of reaching lasting viral control with darunavir-based combined salvage therapy.

In our survey, the sample point estimate of the prevalence rate of patients infected with HIV with 3 or more DRV-RAM was $6.7 \%$. Interestingly, the frequency of darunavir resistance was significantly higher in our study population when compared with a rate of $4.1 \%$ (a value which is not within the boundaries of the $95 \%$ confidence interval of our estimate) reported in a population of PItreated patients in 16 clinics of the Kaiser-Permanente Medical Care Program in Northern California [10], despite an equal median number of PIs administered in both surveys.

The determinants of successful DRV/r-based therapy in highly antiretroviral-experienced HIV-infected patients, have been identified. In these patients, virologic success is independently associated with fewer major PI mutations and the use of new drug classes and/or fully active drugs in the salvage regimen [15]. Moreover, the analysis of pooled 24-week data from POWER 1, 2 and 3 showed that the virologic response was significantly diminished with 3 or more baseline DRV-RAM in the context of a high number of PI-RAM [7].

In comparison to the baseline features of participants in randomized clinical trials testing the efficacy of DRV/ $\mathrm{r}$-containing salvage regimens in treatment-experienced HIV patients (POWER 1 and 2 and DUET 1 and 2) [2, $4,5]$ subjects in our study were characterized by a relatively lower prior exposure to PI (median of 2 drugs, median cumulative time of PI usage of 5 years), a lower resistance profile to PIs (median of 2 major PI-RAM) and a significantly lower prevalence of DRV-resistant HIV. Thus, assuming that patients in our survey will be highly adherent to the prescribed DRV/r-based salvage therapy, combined with at least 2 other novel and/or fully active drugs, a higher rate (compared to that of those trials) of a lasting plasma viral load under the limit of detection is to be expected. In contrast, patients with at least one major PI-RAM (which occurred in $70 \%$ of our patients) would be less likely to achieve this goal with lopinavir/ritona- 
vir-containing salvage combined therapy [16].

Our data show that the number of major PI-RAM detected by genotype testing is an accurate proxy of the magnitude of prior PI exposure/failure and of pharmacologic PI selective pressure during long periods of treatment with PI-containing failing regimens. Our findings provide further support to the concept that the risk of infection with darunavir-resistant HIV is directly related to the level and duration of previous PI exposure; there is a 3.7 fold increase in this risk per each additional major PIRAM identified by genotype testing. We also found that specific non-darunavir PI-RAM (such as M46I, Q58E, V82T and L90M) were associated with darunavir resistance, as was also shown by Mitsuya et al. [10].

By multivariate analysis, we were able to establish that previous use of (fos) amprenavir and/or tipranavir significantly increases the risk of darunavir resistance, independently of the number of major PI-RAM (as an indicator of the prior overall load of PI selective pressure). Both molecules, (fos) amprenavir and darunavir, are structurally related and hence, share some specific resistance mutations [17]. In our study sample, this effect of (fos) amprenavir might be mediated through the selection of mutations I50V and I54M which were more common in individuals who had received these agents versus other PI.

Interestingly, to our knowledge this is the first report of the previous use of tipranavir as an independent risk factor for the loss of viral susceptibility to darunavir. A possible explanation of this finding is that a failing tipranavir-containing regimen increases the likelihood of selecting certain PI-RAM conferring cross-resistance with darunavir (such as mutations L33F, I47V, T74P and I84V). During the last six years, the prescription of this PI in Mexico has been unrestricted and commonly used inappropriately since it has been added to other antiretroviral agents without full antiviral activity (functional monotherapy); we are now seeing the deleterious effect of this erroneous clinical practice on darunavir-viral susceptibility.

A potential methodological drawback of this study is the possible lack of representativeness of our study sample (sample selection bias) which may limit the extrapolation of our results to other multi-drug exposed patients but with a different degree of previous PI use. The prevalence of DRV-RAM-bearing HIV needs to be determined in other populations with a diverse history of PI selective pressure.

\section{Conclusion}

In summary, in this sample of heavily antiretroviral treated patients, despite the fact that most had a history of several years under selective pressure by PI-including failing regimens, the emergence of HIV variants with DRV-RAM was of relatively low magnitude. DRV/r- based salvage therapy with at least 2 other novel and/or fully active drugs is likely to be successful in a significant proportion of these patients, with expected higher rates of virologic control compared to those found in clinical trial participants. Future similar surveys are necessary in order to continuously monitor this dynamic phenomenon; a rise in the incidence of darunavir-resistant HIV infections is likely to occur with longer patient survival and an increase in overall PI (including tipranavir and darunavir) exposure.

\section{Source of Research Funds}

The Inter-institutional group for antiretroviral treatment (GUIAR) group was funded with a grant provided by Merck Sharp \& Dohme and The Board for the rational use of antiretrovirals (CORESAR), with subsidies provided by the Centro Nacional para la Prevención y el Control del VIH/SIDA (CENSIDA-México).

\section{Acknowledgements}

We thank Dr. Wendy K. Moncada, Dr. Yukie GarcíaKishi, Dr. Elsa Vidal-Laurencio and Luis Fuentes-Romero, BSc, from the Department of Infectious Diseases, Instituto Nacional de Ciencias Médicas y Nutrición Salvador Zubirán, Mexico City, Mexico, for their technical assistance. We are particularly grateful to the physicians who referred clinical cases for peer-advising on salvage antiretroviral therapy.

\section{REFERENCES}

[1] A. Imaz, V. Falcó and E. Ribera, "Antiretroviral Salvage Therapy for Multiclass Drug-Resistant HIV-1-Infected Patients: From Clinical Trials to Daily Clinical Practice," AIDS, Vol. 13, 2011, pp. 180-193.

[2] B. Clotet, N. Bellos, J. M. Molina, et al., "Efficacy and Safety of Darunavir-ritonavir at Week 48 in TreatmentExperienced Patients with HIV-1 Infection in POWER 1 and 2: A Pooled Subgroup Analysis of Data from Two Randomized Trials," Lancet, Vol. 369, No. 9568, 2007, pp. 1169-1178. doi:10.1016/S0140-6736(07)60497-8

[3] K. Arastéh, P. Yeni, A. Pozniak, et al., "Efficacy and Safety of Darunavir/Ritonavir in Treatment-Experienced HIV Type-1 Patients in the POWER 1, 2 and 3 Trials at Week 96," Antiviral Therapy, Vol. 14, 2009, pp. 859-864. doi:10.3851/IMP1301

[4] J. V. Madruga, P. Cahn, B. Grinztejn, et al., "Efficacy and Safety of TMC125 (Etravirine) in Treatment-Experienced HIV-1-Infected Patients in DUET-1: 24-Week Results from a Randomized, Double Blind, Placebo-Controlled Trial," Lancet, Vol. 370, No. 9581, 2007, pp. 29-38. doi:10.1016/S0140-6736(07)61047-2

[5] A. Lazzarin, T. Campell, B. Clotet, et al., "Efficacy and Safety of TMC125 (Etravirine) in Treatment-Experienced HIV-1-Infected Patients in DUET-2: 24-Week Results 
from a Randomized, Double Blind, Placebo-Controlled Trial," Lancet, Vol. 370, No. 9581, 2007, pp. 39-48. doi:10.1016/S0140-6736(07)61048-4

[6] C. Katlama, B. Clotet, A. Mills, et al., "Efficacy and Safety of Etravirine at Week 96 in Treatment-Experienced HIV Type-1-Infected Patients in the DUET-1 and DUET2 Trials," Antiviral Therapy, Vol. 15, 2010, pp. 10451052. doi:10.3851/IMP1662

[7] S. De Meyer, T. Vangeneugden, B. van Baelen, et al., "Resistance Profile of Darunavir: Combined 24-Week Results from the POWER Trials," AIDS Research and Human Retroviruses, Vol. 24, No. 3, 2008, pp. 379-388. doi:10.1089/aid.2007.0173

[8] J. E. Vidal, A. C. Freitas, A. T. W. Song, S. V. Campos, M. Dalben and A. V. Hernandez, "Prevalence and Factors Associated with Darunavir Resistance Mutations in Multi-Experienced HIV-1-Infected Patients Failing Other Protease Inhibitors in a Referral Teaching Center in Brazil," Brazilian Journal of Infectious Diseases, Vol. 15, No. 3, 2011, pp. 245-248. doi:10.1016/S1413-8670(11)70183-0

[9] E. Poveda, C. de Mendoza, L. Martin-Carbonero, et al., "Prevalence of Darunavir Resistance Mutations in HIV-1Infected Patients Failing Other Protease Inhibitors," Journal of Antimicrobial Chemotherapy, Vol. 60, No. 4, 2007, pp. 885-888. doi:10.1093/jac/dkm276

[10] Y. Mitsuya, T. F. Liu, S. Y. Rhee, W. J. Fessel and R. W. Shafer, "Prevalence of Darunavir Resistance-Associated Mutations: Patterns of Occurrence and Association with Past Treatment," The Journal of Infectious Diseases, Vol. 196, No. 8, 2007, pp. 1177-1179. doi:10.1086/521624

[11] S. Bautista-Arredondo, A. Mane and S. M. Bertozzi, "Economic Impact of Antiretroviral Therapy Prescription Decisions in the Context of Rapid Scaling-Up of Access to Treatment: Lessons from Mexico," AIDS, Vol. 20, No. 1,
2006, pp. 101-109. doi:10.1097/01.aids.0000198096.08444.53

[12] J. J. Calva and Y. Vargas-Infante, "Cobertura Universal con la Terapia Antirretroviral Combinada. Logros y Desafíos en la Secretaría de Salud de Mexico," In: J. A. Cordova-Villalobos, S. Ponce de Leon-Rosales and J. L. Valdespino, Eds., 25 años de SIDA en Mexico. Logros, Desaciertos y Retos, 2nd Edition, Instituto Nacional de Salud Pública, Cuernavaca, Morelos, 2009, pp. 333-353.

[13] V. A. Johnson, V. Calvez, H. F. Gunthard, et al., "2011 Update of the Drug Resistance Mutations in HIV-1," Topics in Antiviral Medicine, Vol. 19, No. 4, 2011, pp. 156-164.

[14] L. E. Wilson and J. E. Gallant, "The Management of Treatment-Experienced HIV-Infected Patients: New Drugs and Drug Combinations," Clinical Infectious Diseases, Vol. 48, 2009, pp. 214-221.

[15] C. Delaugerre, J. F. Buyck, G. Peytavin, et al., "Factors Predictive of Successful Darunavir/Ritonavir-Based Therapy in Highly Antiretroviral-Experienced HIV-1-Infected Patients (the DARWEST Study)," Journal of Clinical Virology, Vol. 47, No. 3, 2010, pp. 248-252. doi:10.1016/i.jcv.2009.12.022

[16] S. De Meyer, A. Hill, G. Picchio, R. DeMasi, E. De Paepe and M. P. de Bethune, "Influence of Baseline Protease Inhibitor Resistance on the Efficacy of Darunavir/ ritonavir or Lopinavir/Ritonavir in the TITAN Trial," Journal of Acquired Immune Deficiency Syndromes, Vol. 49, No. 5, 2008, pp. 563-564. doi:10.1097/QAI.0b013e318183ac9c

[17] S. Y. Rhee, J. Taylor, W. J. Fessel, et al., "HIV-1 Protease Mutations and Protease Inhibitor Cross-Resistance," Antimicrobial Agents and Chemotherapy, Vol. 54, No. 10, 2010, pp. 4253-4261. 